\title{
Do light nuclei exhibit "collective" motions?
}

\author{
Winfried Leidemann ${ }^{1,2, a}$ \\ ${ }^{1}$ Dipartimento di Fisica, Università di Trento, I-38123 Trento, Italy \\ ${ }^{2}$ Istituto Nazionale di Fisica Nucleare, Gruppo Collegato di Trento, I-38123 Trento, Italy
}

\begin{abstract}
The Lorentz integral transform (LIT) method has allowed to perform ab initio calculations of the response function of ${ }^{4,6} \mathrm{He}$, and ${ }^{6,7} \mathrm{Li}$ to the isoscalar monopole and isovector dipole operators in a wide range of energies. In this work we discuss some of these results and focus in particular on the ${ }^{4} \mathrm{He}$ case, where one has a $0^{+}$resonance close to the $3+1$ thresholds. In fact, in inelastic electron scattering off ${ }^{4} \mathrm{He}$ one finds a pronounced resonant structure in the isoscalar monopole strength. The knowledge of this strength as a function of energy makes possible calculations of the corresponding nonenergy weighted and energy-weighted sum rules. Comparing the sum rules with their contribution from the resonance region hints to the degree of collectivity of the resonant structure.
\end{abstract}

\section{Introduction}

Resonant structures in observables are particularly interesting, since they reveal certain aspects of the dynamics of a particle system. A celebrated example is the giant dipole resonance, which originates from a collective relative motion of neutrons against protons. As will be discussed in the following one may use integral transforms to study such resonance phenomena. In fact, the use of integral transforms is quite common in physics. Typically they have the following form

$$
\Phi(\sigma)=\int d E K(E, \sigma) R(E),
$$

where $R(E)$ is an observable depending on the energy $E$ and $K(E, \sigma)$ is a well defined kernel. The interesting point is that there are cases where it is considerably easier to calculate the integral transform $\Phi(\sigma)$ than the observable $R(E)$. In such a situation one can determines $R(E)$ from the inversion of the integral transform. Here the Lorentz integral transform (LIT) $L(\sigma)[1,2]$ is considered. The LIT is defined by a kernel of Lorentzian shape,

$$
K(E, \sigma)=\frac{1}{\left(E-\sigma_{R}\right)^{2}+\sigma_{I}^{2}}
$$

with $\sigma=\sigma_{R}+i \sigma_{I}$. A calculation of the $L(\sigma)$ can be performed by solving an equation of the form

$$
(H-\sigma) \tilde{\Psi}=S,
$$

\footnotetext{
a e-mail: leideman@science.unitn.it
} 
where $H$ is the Hamiltonian of the system under consideration and $S$ is an asymptotically vanishing source term. The solution $\tilde{\Psi}$ is localized and can be determined applying bound-state methods. The solution leads directly to the LIT:

$$
L(\sigma)=\langle\tilde{\Psi} \mid \tilde{\Psi}\rangle .
$$

It is important to note that the energy $E$ can lie far in the many-body continuum and nonetheless one can carry out rigorous ab initio calculations with the LIT method. In fact a full inclusion of the final state interaction is guaranteed by the method. In the past such LIT calculations have been performed for quite a number of electroweak break-up reactions of few-body nuclei (nucleon number up to $A=7)$. An overview can be found in the reviews [2, 3]. In figure 1 an example is given for the ${ }^{3} \mathrm{He}$ inelastic transverse response function $R_{T}(q, \omega)$ in inclusive $\left(e, e^{\prime}\right)$ scattering [4]. The LIT calculation was carried out using as nuclear interaction the AV18 NN potential [5] and the UIX three-nucleon force (3NF) [6] and considering a non-relativistic nucleon one-body current with additional relativistic corrections, meson exchange currents, and currents involving the $\Delta$ resonance. The employed boundstate method consisted in an expansion in correlated hyperspherical harmonics. From the figure it is evident that there is a very nice agreement between theory and experiment up into the far continuum. Experimental and theoretical results start to be different with the onset of pion production which, however, was not included in the theoretical calculation.

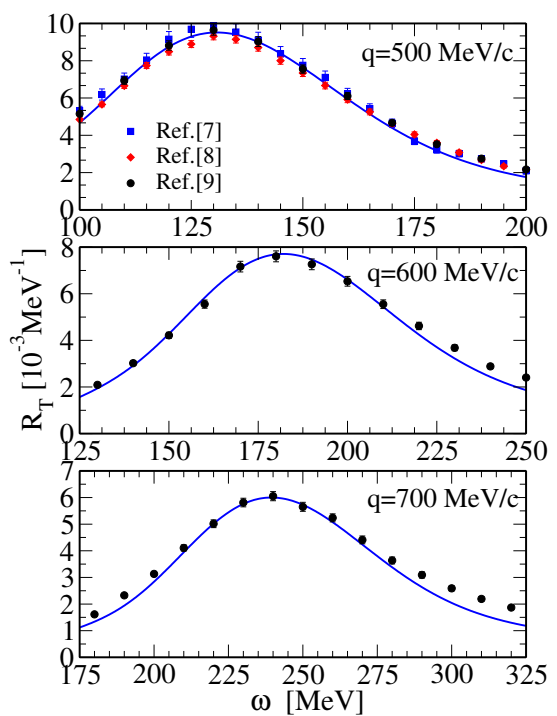

Figure 1. Inelastic transverse response function $R_{T}(q, \omega)$ of ${ }^{3} \mathrm{He}$

\section{LIT approach: a simple example}

Resonances can have a very narrow width. Therefore it is important to understand if they can be resolved with the LIT approach. In order to address this issue a simple example is discussed, namely deuteron photodisintegration in unretarded dipole approximation. In this case the total photoabsorption cross section is given by

$$
\sigma(\omega)=4 \pi^{2} \alpha \omega R(\omega)
$$


INPC 2013

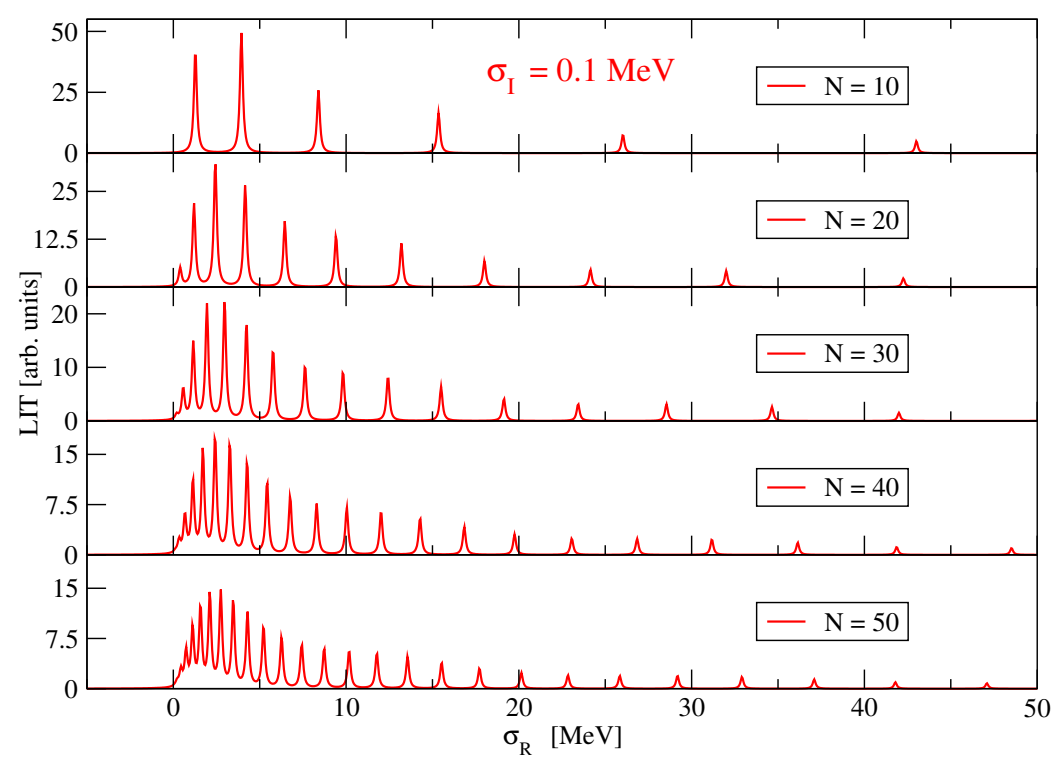

Figure 2. Deuteron photodisintegration in unretarded dipole approximation: LIT result for partial wave ${ }^{3} P_{1}$ and $\sigma_{I}=0.1 \mathrm{MeV}$

with the dipole response function

$$
R(\omega)=\int d f|\langle f|\theta| 0\rangle|^{2} \delta\left(\omega-E_{f}-E_{0}\right),
$$

where $E_{0}, E_{f}$ and $|0\rangle,|f\rangle$ are ground and final state energies and wave functions, respectively, and $\theta=\sum_{i} z_{i} \tau_{i, z} / 2$ is the isovector dipole operator $\left(z_{i}\right.$ and $\tau_{i, z}$ are z-components of position vector and isospin operator of the $\mathrm{i}$-th nucleon). The resulting LIT equation reads

$$
\left(H-E_{0}-\sigma\right)|\tilde{\Psi}\rangle=\theta|0\rangle .
$$

For the deuteron case it is sufficient to make the following ansatz for $\tilde{\Psi}$ :

$$
|\tilde{\Psi}\rangle=\sum_{j=0}^{2} \sum_{l} \tilde{\psi}_{l j}(r)|(l, S=1) j\rangle|T=1\rangle,
$$

where $r(T=1)$ is relative distance (isospin) of the $n p$ pair. This then leads to three separate LIT equations, two uncoupled $\left({ }^{3} P_{0},{ }^{3} P_{1}\right)$ and one coupled case $\left({ }^{3} P_{2}-{ }^{3} F_{2}\right)$. They can be easily solved by direct numerical methods or by expansions of $\tilde{\psi}_{l j}$ on a complete set. Since in case of nuclei with $A>2$ we are generally using expansions on hyperspherical harmonics, where the hyperradial part is expanded on Laguerre polynomials $L_{n}^{m+\frac{1}{2}}$ times an exponential fall-off, we make here a corresponding ansatz,

$$
\tilde{\psi}_{l j}(r)=\sum_{n=1}^{N} c_{n l} r^{l} L_{n}^{l+\frac{1}{2}}(r / b) \exp (-r / 2 b),
$$

where $c_{n l}$ is a normalization factor and $b$ a constant. In figures 2-4 LIT results for the ${ }^{3} P_{1}$ channel are shown for various values of $N$ and $\sigma_{I}(b=0.5 \mathrm{fm})$. One may realize that the results are identical to 
EPJ Web of Conferences

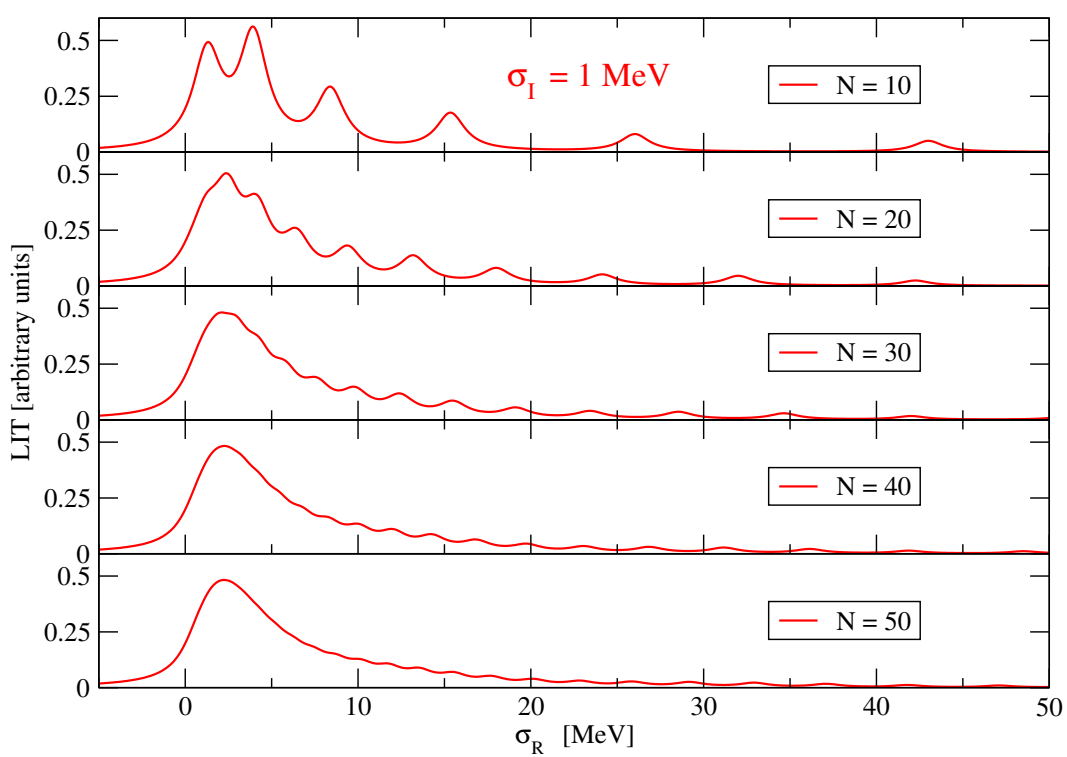

Figure 3. As figure 2 but for $\sigma_{I}=1 \mathrm{MeV}$

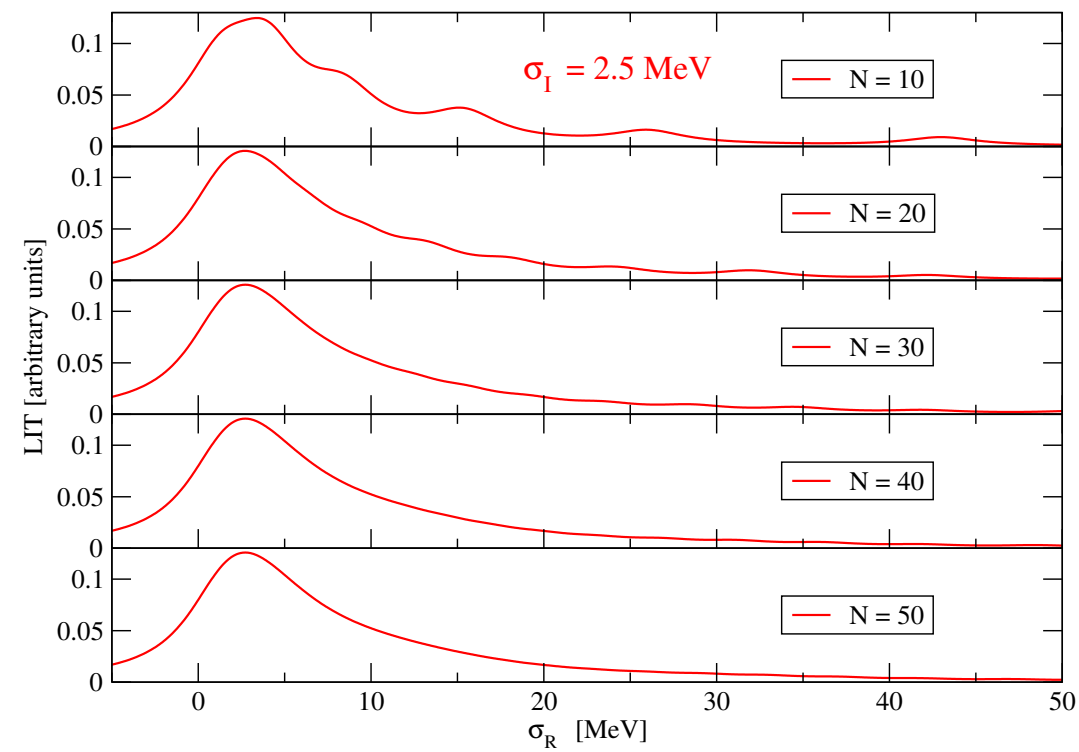

Figure 4. As figure 2 but for $\sigma_{I}=2.5 \mathrm{MeV}$

so-called Lanczos responses $R_{\text {Lanczos }}$ which have the form

$$
R_{\text {Lanczos }}=\sum_{n=1}^{N} \frac{f_{n}}{\left(E_{n}-\sigma_{R}\right)^{2}+\sigma_{I}^{2}}
$$


(for a discussion of LIT method and Lanczos response see [2]). To make a reliable inversion it is necessary to have a sufficiently converged LIT result for a given $\sigma_{I}$. For $\sigma_{I}=0.1 \mathrm{MeV}$ (see figure 2) this is certainly not the case. The number $N$ of Laguerre polynomials had to be increased much more in order to have a converged transform. For the $\sigma_{I}=1 \mathrm{MeV}$ case (see figure 3 ) the situation is already much better. One sees that for $N=50$ a converged result is obtained up to about $10 \mathrm{MeV}$. Thus in this energy range a resolution of at least $1 \mathrm{MeV}$ is guaranteed, i.e. possible structures with a width of $1 \mathrm{MeV}$ should be reliably reproduced by the inversion. As figure 4 shows increasing $\sigma_{I}$ to 2.5 $\mathrm{MeV}$ leads to a converged LIT in the whole considered energy range with $N=50$. From the results from figures 2-4 it is evident that $\sigma_{I}$ is strongly correlated to the convergence of the expansion. On the other hand is not surprising that one has to increase $N$ in order to work with a higher resolution. In contrast to other integral transform, e.g., the Laplace transform, the LIT has the nice feature that it is a method with a controlled resolution. In fact one is able to check up to which value of $\sigma_{I}$ a converged result is obtained. This is a very important aspect, since one has a criterion that allows one to decide whether a certain structure in the inversion result is real or fake. This makes the inversion very reliable. For a discussion of the inversion procedure itself the reader is referred to [2, 10], but it should be pointed out, that even resonances with a width as small as $300 \mathrm{keV}$ can be reliably resolved with the LIT method [11].

\section{LIT method and resonances}

As discussed in Sect. 2 one must be able to calculate a converged LIT for a sufficiently small $\sigma_{I}$ in order to resolve the detailed structure of a resonance. In the following we will consider one of the most recent LIT application, namely the isoscalar monopole resonance of ${ }^{4} \mathrm{He}$, but before we illustrate briefly LIT results for the giant dipole resonance (GDR) of light nuclei in unretarded dipole approximation. All the various calculations we are discussing in this section have been performed using expansions of nuclear ground and LIT states in hyperspherical harmonics with the effective interaction approach (EIHH [12]).

\subsection{Giant dipole resonance}

One of the very first LIT calculations was performed for the GDR of ${ }^{4} \mathrm{He}$ [13]. Simple semi-realistic NN potential models were used. A rather pronounced GDR was obtained, considerably higher than the at the time most recent experimental results. Later the pronounced GDR of ${ }^{4} \mathrm{He}$ was confirmed in a second LIT calculation [14] with a realistic nuclear interaction (AV18 NN potential and UIX 3NF). Also on the experimental side new measurements were performed which confirm the pronounced GDR, however the experimental situation is not yet completely settled (see $[3,15])$.

LIT calculations for the GDR of ${ }^{6} \mathrm{He},{ }^{6} \mathrm{Li}$, and ${ }^{7} \mathrm{Li}[16-18]$ were carried out with semi-realistic potential models. On the experimental side the ${ }^{6} \mathrm{He}$ and ${ }^{6} \mathrm{Li}$ cases are quite involved, whereas one has a rather clear picture for the ${ }^{7} \mathrm{Li}$ total photoabsorption cross section and the comparison with the LIT result is quite satisfying. On the other hand the theoretical cross section for the unstable nucleus of ${ }^{6} \mathrm{He}$ is very interesting. Different from the other cases it exhibits a double peak structure. One finds a low-lying rather narrow peak and a second rather broad peak beyond $20 \mathrm{MeV}$. The second peak should correspond to the GDR, while the low-energy peak might be interpreted as a relative motion of the two outer neutrons relative to the $\alpha$-core.

Very recently a LIT calculation for the GDR of ${ }^{16} \mathrm{O}$ was carried out. For results I refer the reader to $\mathrm{S}$. Bacca's contribution in the plenary session. 


\subsection{Isoscalar monopole resonance of ${ }^{4} \mathrm{He}$}

The ${ }^{4} \mathrm{He}$ inclusive longitudinal response function $R_{L}(q, \omega)$ in inelastic $\left(e, e^{\prime}\right)$ scattering shows quite interesting features. This response functions exhibits a strong influence of the $3 \mathrm{NF}$ at low energy and momentum transfers up to $q=200 \mathrm{MeV} / \mathrm{c}[19,20]$. The $3 \mathrm{NF}$ effect can reach almost $50 \%$ and depends also on the choice of the $3 \mathrm{NF}$ itself. A further aspect of the $R_{L}$ of ${ }^{4} \mathrm{He}$ is the presence of an

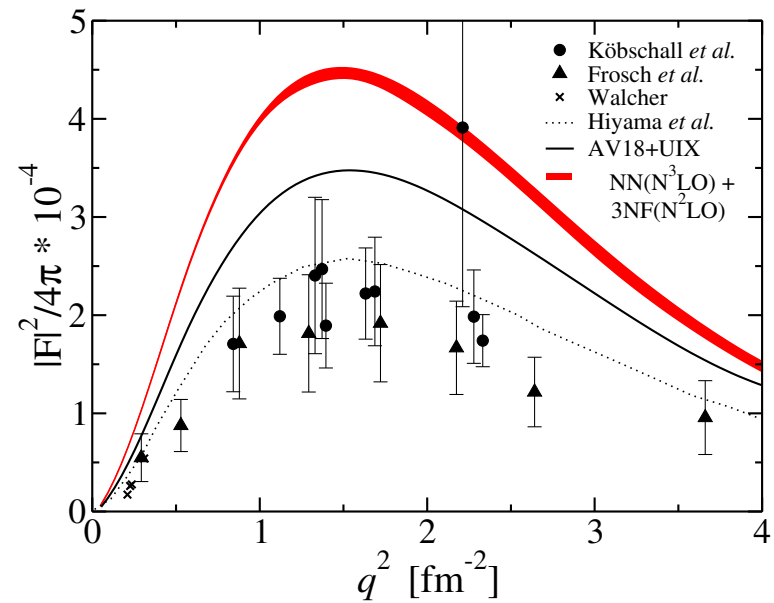

Figure 5. ${ }^{4} \mathrm{He}$ isoscalar monopole resonance strength $\left|F\left(q^{2}\right)\right|^{2}$

isoscalar monopole resonance, which is located between the two thresholds for the break-up in ${ }^{3} \mathrm{H}-p$ and ${ }^{3} \mathrm{He}-n$. The resonance leads to a pronounced cross section peak in inelastic electron scattering as has been observed in various experiments [21-23]. The width of the resonance was determined to be about $300 \mathrm{keV}$.

In [24] the isoscalar monopole part of $R_{L}$ was calculated with the LIT method. Thanks to the fact that the LIT is a method with controlled resolution and since a converged LIT result could only be obtained for $\sigma_{I} \geq 5 \mathrm{MeV}$ it was clear beforehand that it was not possible to disentangle the detailed structure of the resonance. As described in [24] it was nonetheless possible to determine the resonance strength and the energy dependence of the background strength by a proper inversion procedure. It should be noted that it is not sufficient to identify as resonance strength the strength $f_{n_{0}}$ of $R_{\text {Lanczos }}$ (see Eq. 10), where $E_{n_{0}}$ is the resonance energy. The reason is that $f_{n_{0}}$ contains also background strength (see corresponding discussion in [24]).

The calculation [24] was carried out with two different nuclear interaction (AV18 NN potential and UIX 3NF, Idaho-N3LO NN potential [25] and an N2LO 3NF in two slightly different parameterizations). Both interaction models overestimate the resonance position by about $700 \mathrm{keV}$. Results for the strength of the resonance are shown in figure 5. Particularly striking is the strong dependence of the results on the interaction model, note that both of them represent realistic nuclear forces. In addition it is very surprising that the experimental strength is considerably smaller than that of the theory, especially for the chiral potential model. As discussed in detail in [24] it is not easy to understand what causes the difference of theoretical and experimental results (e.g., the calculated elastic ${ }^{4} \mathrm{He}$ form factors for both interaction models agree well with experiment up to about $q^{2}=4 \mathrm{fm}^{-2}$. In figure 5 also a result from [26] is shown, which agrees much better with the experimental data. The calculation, however, cannot be considered to be fully realistic. The employed interaction model consists in the AV8' $\mathrm{NN}$ potential model and a simplistic $3 \mathrm{NF}$, and in addition the excited $0^{+}$state is 
INPC 2013

Table 1. Relative contribution of resonance strength $|F(q)|^{2}$ to $m_{0}$ and $m_{1}$ sum rules

\begin{tabular}{lll}
\hline$q[\mathrm{MeV} / \mathrm{c}]$ & $|F(q)|^{2} / m_{0}[\%]$ & $E_{R}|F(q)|^{2} / m_{1}[\%]$ \\
\hline 50 & 50 & 26 \\
100 & 45 & 29 \\
150 & 39 & 24 \\
200 & 32 & 18 \\
300 & 20 & 10 \\
\hline
\end{tabular}

considered to be a bound state. All this shows that further investigations are necessary to understand the underlying physics better.

\section{Sum rules and collectivity}

The example of the GDR shows that sum rules may serve to judge the collectivity of a specific resonance. In fact, all over the nuclear table the GDR exhausts the by far greatest part of the electric dipole sum rule. This indicates that a collective motion is occurring in the resonance region, since otherwise one would expect a spread of strength over a larger energy range.

Here we consider the non-energy weighted and the energy weighted sum rules for the ${ }^{4} \mathrm{He}$ isoscalar monopole strength $F_{0}^{\mathrm{IS}}(q, E)$,

$$
\begin{aligned}
& m_{0}(q)=\int d E F_{0}^{\mathrm{IS}}(q, E)=\left\langle 0\left|\rho_{M}^{\dagger}(q) \rho_{M}(q)\right| 0\right\rangle \\
& m_{1}(q)=\int d E E F_{0}^{\mathrm{IS}}(q, E)=\left\langle 0\left|\rho_{M}^{\dagger}(q) H \rho_{M}(q)\right| 0\right\rangle=\frac{1}{2}\left\langle 0\left|\left[\rho_{M}^{\dagger}(q),\left[H, \rho_{M}(q)\right]\right]\right| 0\right\rangle,
\end{aligned}
$$

where $\rho_{M}(q)$ is the isoscalar monopole part of the nuclear charge operator. In the limit $q \rightarrow 0$ one has $\rho_{M}(q) \rightarrow q^{2} \sum_{i} r_{i}^{2}$ and thus one finds $m_{1}(q) \rightarrow A q^{2}\left\langle r^{2}\right\rangle / m_{N}$, where $m_{N}$ is the nucleon mass. Setting $\left\langle r^{2}\right\rangle$ to the experimental value one has a model independent result for $m_{1}(q)$ in the limit $q \rightarrow 0$. With a calculation of the resonance strength in the limit $q \rightarrow 0$ one can check how much the resonance exhausts the sum rule. If the exhaustion is sufficiently large one may speculate about the existence of a collective motion. However, such a strategy has a drawback. In fact if only a small part of the strength is located at higher energies it will give a considerable contribution to the energy weighted sum rule $m_{1}$. It seems to be better to use the non-energy weighted sum rule $m_{0}$ to check the collectivity, since in this case a small high-energy strength would not affect the picture, but on the other hand one has to be able to determine the model dependent result for $m_{0}$. Our LIT calculation discussed in Sect. 3.2 easily allows such a determination. Results for the exhaustion of $m_{0}$ and $m_{1}$ sum rules are listed in table 1 . One sees that in case of $m_{1}$ one has a maximal exhaustion of $29 \%$ at $q=100 \mathrm{MeV} / \mathrm{c}$, whereas in case of $m_{0}$ one reaches a maximal exhaustion of $50 \%$ for the $q$-value of $50 \mathrm{MeV} / \mathrm{c}$. The trend of the results indicates that the exhaustion could become even larger at $q<50 \mathrm{MeV} / \mathrm{c}$. Particularly, the $m_{0}$ result leaves certainly space for a speculation of the existence of a collective effect in the ${ }^{4} \mathrm{He}$ isoscalar monopole resonance. However, further calculations should be performed in order to confirm such a collectivity. For example, one could study the transition density.

\section{Summary}

The LIT method has been applied to study resonance phenomena in light nuclei. It has been shown that the LIT approach is a method with a controlled resolution. This is an important aspect, since one 


\section{EPJ Web of Conferences}

has a criterion at disposal to decide to what extent a resonant structure can be reliably obtained in the inversion.

Various examples for the GDR of light nuclei have been discussed and the GDR of ${ }^{6} \mathrm{He}$ is certainly the most interesting of these cases. Concerning the ${ }^{4} \mathrm{He}$ isoscalar monopole strength one finds quite puzzling results. In fact one has rather different results with two different realistic nuclear interaction models, which, in addition overestimate the experimental results considerably. It has also been discussed that sum rule arguments allow one to speculate that collective effects could play a role for the dynamics of the $0^{+}$resonance of ${ }^{4} \mathrm{He}$. Thus I conclude that the study of the physics of the ${ }^{4} \mathrm{He}$ resonance is very exciting and that many aspects need still to be investigated much better.

\section{References}

[1] V.D. Efros, W. Leidemann, and G. Orlandini, Phys. Lett. B 338, 130 (1994)

[2] V.D. Efros, W. Leidemann, G. Orlandini, and N. Barnea, J. Phys. G 34, R459 (2007)

[3] W. Leidemann and G. Orlandini, Progr. Part. Nucl. Phys. 68, 158 (2013)

[4] L. Yuan, W. Leidemann, V.D. Efros, G. Orlandini and E.L. Tomusiak, Phys. Lett. B 706, 90 (2011)

[5] R. B. Wiringa, V. G. J. Stoks, and R. Schiavilla, Phys. Rev. C 51, 38 (1995)

[6] B.S. Pudliner, V.R. Pandharipande, J. Carlson, S.C. Pieper, and R.B. Wiringa, Phys. Rev. C 56, 1720 (1997)

[7] C. Marchand et al., Phys. Lett. B 153, 29 (1985)

[8] K. Dow et al., Phys. Rev. Lett. 61, 1706 (1988)

[9] J. Carlson, J. Jourdan, R. Schiavilla, and I. Sick, Phys. Rev. C 65, 024002 (2002)

[10] D. Andreasi, W. Leidemann, Ch. Reiss and M. Schwamb, Eur. Phys. J. A, 24, 361 (2005)

[11] W. Leidemann, Few-Body Syst. 42, 139 (2008)

[12] N. Barnea, W. Leidemann, and G. Orlandini, Phys. Rev. C 61, 54001 (2000); Nucl. Phys. A 693, $565(2001)$

[13] V.D. Efros, W. Leidemann, and G. Orlandini, Phys. Rev. Lett. 78, 4015 (1997)

[14] D. Gazit, S. Bacca, N. Barnea, W. Leidemann and G. Orlandini, Phys. Rev. Lett. 96, 112301 (2006)

[15] R. Raut et al., Phys. Rev. Lett. 108, 042502 (2012)

[16] S. Bacca, M.A. Marchisio, N. Barnea, W. Leidemann, and G. Orlandini, Phys. Rev. Lett. 89, 052502 (2002)

[17] S. Bacca, N. Barnea, W. Leidemann, and G. Orlandini, Phys. Rev. C 69, 57001 (2004)

[18] S. Bacca, H. Arenhövel, N. Barnea, W. Leidemann and G. Orlandini, Phys.Lett. B 603, 159 (2004)

[19] S. Bacca, N. Barnea, W. Leidemann, and G. Orlandini, Phys. Rev. Lett. 102, 162501 (2009)

[20] S. Bacca, N. Barnea, W. Leidemann, and G. Orlandini, Phys. Rev. C 80, 064001 (2009)

[21] Th. Walcher, Phys. Lett. B, 31, 442 (1970)

[22] R.F. Frosch et al., Phys. Lett. 19, 155 (1965); Nucl. Phys. A, 110, 657 (1968)

[23] G. Köbschall et al., Nucl. Phys. A, 405, 648 (1983)

[24] S. Bacca, N. Barnea, W. Leidemann, and G. Orlandini, Phys. Rev. Lett. 110, 042503 (2013)

[25] D.R. Entem and R. Machleidt, Phys. Rev. C 68, 041001 (2003)

[26] E. Hiyama, B.F. Gibson, and M. Kamimura, Phys. Rev. C 70, 031001 (2004) 